\title{
Jamestown Canyon virus meningoencephalitis mimicking migraine with aura in a resident of Manitoba
}

\author{
Reza Vosoughi MD, Andrew Walkty MD, Michael A. Drebot PhD, Kamran Kadkhoda PhD
}

Cite as: CMAJ 2018 March 5;190:E262-4. doi: 10.1503/cmaj.170940

A 26-year-old man with no substantial medical history presented to an academic emergency department in Winnipeg with a headache and associated neurologic symptoms. The patient reported that earlier in the day while driving a car, he had felt a sudden tingling and numbness in his right foot. This subsequently spread up the right side of his body to his right leg, right arm, and the right side of his face. The episode lasted about 10 minutes before the symptoms spontaneously resolved. Later the same day, a second episode of rightsided numbness occurred. This time, the symptoms were associated with difficulty speaking, prompting the patient to go to the emergency department.

Between 45 and 60 minutes after the onset of symptoms, the patient had a moderate-to-severe throbbing headache. The speech and sensory problems resolved within 2 hours and 30 minutes, whereas the headache improved within 11 hours. The patient reported no history of migraine headaches. During the visit to the emergency department, computed tomography (CT) imaging of the brain, $\mathrm{CT}$ angiogram of the cervical and cranial arteries and unenhanced magnetic resonance imaging (MRI) of the brain done with diffusion weighted imaging and apparent diffusion coefficient mapping were all unremarkable. The patient returned home the same day with a diagnosis of migraine with aura.

Two days later, the patient woke up early in the morning with numbness and tingling in his left foot, which gradually spread over several minutes to his left arm and the left side of his face, and was associated with a headache and a milder degree of speech impairment in the form of word-finding difficulty. No visual symptoms were reported. The patient again presented to the emergency department for further evaluation. Within 1 hour his neurologic deficits had improved, but he continued to complain of a severe bifrontal throbbing headache, with associated nausea, vomiting, photophobia and phonophobia. The headache was worse with standing and activity. The patient reported no fever, chills, sweats, neck stiffness or rash. A review of systems was otherwise negative. The patient lived in Winnipeg and reported no recent travel outside of Manitoba. He had no contact with animals or people who were ill. There was no history of tick exposure. However, the patient did report numerous recent mosquito bites.

\section{KEY POINTS}

- Jamestown Canyon virus, a California serogroup virus, is widely distributed across Canada.

- Transmission to humans occurs through the bite of an infected mosquito.

- Most patients exposed to the virus have no symptoms, but infection can result in a nonspecific febrile illness or neuroinvasive disease including meningitis and encephalitis.

- Clinicians in Canada should consider California serogroup viruses in the differential diagnosis for patients who present with aseptic meningitis or encephalitis during the mosquito season, particularly when another cause is not readily apparent after initial diagnostic testing.

On physical examination, the patient was afebrile and hemodynamically stable, with a blood pressure of $165 / 97 \mathrm{~mm} \mathrm{Hg}$ and a heart rate of 70 beats/min. Cardiac, respiratory and abdominal examinations were unremarkable. No nuchal rigidity or rash was seen. The neurologic examination showed blurring of the optic disk margins bilaterally. Basic electrolytes, serum creatinine and liver enzymes were within normal limits. The patient had a normal total white blood cell count, hemoglobin and platelet count. Additional CT imaging of the brain was unremarkable. Electroencephalography did not show any epileptiform discharges. A repeat MRI of the brain showed suspected leptomeningeal enhancement over both convexities, and increased cortical T2 signal involving both hemispheres, particularly the left parietal and left posterior temporal lobes. A lumbar puncture was performed. The cerebrospinal fluid (CSF) protein was mildly elevated (0.62 [normal $0.2-0.4] \mathrm{g} / \mathrm{L})$, glucose was normal (3.5 [normal 2.3-4.7] mmol/L, corresponding serum glucose $5.1 \mathrm{mmol} / \mathrm{L})$, the cell count was elevated $\left(88 \times 10^{6}\right.$ [ $89 \%$ lymphocytes; normal 0 to $5 \times 10^{6}$ cells/L), and the opening pressure was $290 \mathrm{~mm} \mathrm{H}_{2} \mathrm{O}$. Overall, these results were thought to be most in keeping with a viral infection or meningoencephalitis, and the patient was admitted to hospital for observation and further investigation.

Serology was negative for Human Immunodeficiency Virus-1 and -2, Mycoplasma pneumoniae immunoglobulin M (IgM), 
Mumps virus IgM, syphilis, Lyme disease, Epstein-Barr virus IgM (virus capsid antigen), West Nile virus IgM and Powassan virus antibody (hemagglutination inhibition testing). Molecular testing by polymerase chain reaction on CSF was negative for Herpes Simplex virus-1 and -2, Varicella zoster virus, Cytomegalovirus and enteroviruses. However, the patient had positive serology for Snowshoe hare virus and Jamestown Canyon virus IgM antibody using an IgM-capture enzyme-linked immunosorbant assay (ELISA) in both serum and CSF specimens collected two and six days after the onset of symptoms, respectively. Neutralizing antibodies to Jamestown Canyon virus were detected in the patient's sera with a titre of more than 80 , but were negative for Snowshoe hare virus by the Jamestown Canyon virus/Snowshoe hare virus plaque reduction neutralization test at the National Microbiology Laboratory in Winnipeg. These results were consistent with an acute neuroinvasive Jamestown Canyon virus infection. The patient's symptoms resolved the day of his second presentation to the emergency department. He had no further episodes of numbness, tingling or headache during his stay in hospital. He was subsequently discharged home five days after admission. When seen for follow-up about two months later, he remained clinically well, with no long-term sequelae related to the infection.

\section{Discussion}

Jamestown Canyon virus is an arthropod-borne virus (arbovirus) belonging to the California serogroup within the genus Orthobunyavirus and Bunyaviridae family of viruses. ${ }^{1}$ Other medically important California serogroup viruses in this genus include Snowshoe hare virus and La Crosse virus. ${ }^{1}$ Jamestown Canyon virus was first isolated in 1961 from a pool of Culiseta inornata mosquitoes collected in Jamestown, Colorado. ${ }^{2}$ It is an enveloped, single-stranded RNA virus with tripartite genome. ${ }^{3}$ In nature, Jamestown Canyon virus is maintained in an enzootic transmission cycle involving amplifying host or reservoir animals and mosquito vectors. ${ }^{1}$ The primary amplifying host animals are white-tailed deer and other free-ranging ungulates, including moose and bison. ${ }^{1,3,4}$ Non-Culex mosquitos (e.g., Aedes, Culiseta, Anopheles) are the main vectors, and the virus can be passed on to progeny by transovarial transmission. ${ }^{1}$ Jamestown Canyon virus is found in the saliva of infected mosquitoes, and transmission to humans (and other animals) may occur when an infected mosquito takes a blood meal. ${ }^{1,3}$

Jamestown Canyon virus appears to be widely distributed throughout the United States, although clinical disease among humans is infrequently documented., ${ }^{1,2,5,6}$ Most recent cases in humans have been reported in the upper midwest and the northeast regions of the United States. ${ }^{2,5}$ In a review of 31 cases of infection with Jamestown Canyon virus by Pastula and colleagues, $42 \%$ of patients were from Wisconsin, although these findings may have been affected by enhanced surveillance efforts there. ${ }^{2}$ Clinical cases have been identified in various provinces across Canada, including British Columbia, Alberta, Saskatchewan, Manitoba, Ontario, Quebec, New Brunswick and Nova Scotia. ${ }^{1,7}$ In 2016, there were 24 laboratory-confirmed Cali- fornia serogroup virus cases or exposures diagnosed by the National Microbiology Laboratory (in comparison with 104 cases of West Nile Virus infection in humans). Jamestown Canyon virus accounted for $82 \%$ of cases where virus classification was done. ${ }^{7}$ Furthermore, recent serologic surveys suggest that exposure to Jamestown Canyon virus among residents in many parts of Canada is not uncommon. ${ }^{3,8-10}$ A study done in Nova Scotia using residual sera obtained from patients for unrelated diagnostic testing showed an overall seroprevalence of $21.2 \%$ for Jamestown Canyon virus. ${ }^{8} \mathrm{~A}$ seroprevalence study conducted among residents of several Cree communities in Quebec showed that $9 \%-24 \%$ of people who underwent testing had serologic evidence of previous Jamestown Canyon virus infection. ${ }^{9}$ Finally, Makowski and colleagues tested a random set of 1366 serum samples from patients in Manitoba who were negative for West Nile virus. ${ }^{10}$ About $10 \%$ of these samples were positive for IgM to California serogroup viruses. ${ }^{10}$ Detection of antibodies to Jamestown Canyon virus among horses, sheep, and cattle in Newfoundland and Labrador provides further support for circulation of this virus in Atlantic Canada. ${ }^{3}$

The incubation period for California serogroup viruses is about three to seven days. ${ }^{4}$ It is thought that most infections with Jamestown Canyon virus are asymptomatic. ${ }^{1}$ The exact ratio of symptomatic to asymptomatic cases is unclear, but it has been estimated to be in the range of $1: 100$ to $1: 1500 .{ }^{1}$ Exposures to California serogroup viruses may occur throughout the mosquito season. ${ }^{2}$ Patients infected with Jamestown Canyon virus may present earlier in the year than those infected with West Nile virus, which relates to the wide variety of mosquito vectors that transmit this pathogen. However, there is most likely an increased risk for exposure during the late summer months when virus circulation may peak in intensity. In two recent publications, most patients with clinical disease were between 20 and 60 years of age, and there was a slight male predominance. ${ }^{2,5}$

Symptomatic infection can present as a nonspecific febrile illness or a neuroinvasive syndrome including meningitis or encephalitis. ${ }^{1,11}$ Meningitis is characterized by fever, headache, neck stiffness and pleocytosis on examination of CSF. ${ }^{1}$ Patients with encephalitis may present with fever, headache and altered mental status. ${ }^{1}$ Acute flaccid paralysis is not commonly reported. ${ }^{5}$ Neuroinvasive disease has been documented in about $55 \%$ of patients in recent case series..$^{2,5}$ However, this likely reflects a bias toward preferential testing in patients presenting with evidence of a central nervous system infection. Treatment of infection is supportive. Death related to Jamestown Canyon virus infection is uncommon. ${ }^{2,5}$ In some cases of encephalitis, long-term neurologic sequelae have been documented, including persisting cognitive deficits. ${ }^{1,12}$

The clinical presentation of this patient's case was unusual, in that it mimicked a migraine with aura. For our patient, the prolonged duration of aura and the finding of papilledema on physical examination were atypical of primary migraine, prompting additional diagnostic testing. We found one previous report in the literature of a patient with Jamestown Canyon virus infection that was initially misdiagnosed as a complex migraine. ${ }^{6}$ That patient presented with severe frontal headache, dizziness and 
left-sided numbness and tingling. However, he also had a fever, providing a clue as to the infectious cause of his symptoms. ${ }^{6}$

Diagnosis of infection with Jamestown Canyon virus is typically made by serology. ${ }^{1}$ Testing is currently offered at the National Microbiology Laboratory. Serum samples are first tested for the presence of Jamestown Canyon virus and Snowshoe hare virus IgM using IgM-capture ELISA, with a turnaround time of about 14 days. ${ }^{1}$ IgM-capture ELISA cross reactions between Jamestown Canyon virus and Snowshoe hare virus are seen for certain California serogroup virus infections. Samples that generate positive or equivocal results are further analyzed for the presence of neutralizing antibodies using the Jamestown Canyon virus and Snowshoe hare virus plaque reduction neutralization test (turnaround time of 14 days from completion of ELISA testing). ${ }^{1}$ Serological testing for IgM using ELISA can be performed on CSF specimens once serum has already tested positive for Jamestown Canyon virus and Snowshoe hare virus IgM in patients presenting with neuroinvasive disease.

A case of Jamestown Canyon virus or Snowshoe hare virus infection is considered "confirmed" by any of the following: a fourfold or greater rise in virus-specific neutralizing antibody titre, the presence of either virus-specific IgM or neutralization antibodies in CSF, detection of virus-specific RNA in CSF or isolation of virus from CSF by cell culture. However, the latter two tests are seldom successful, leaving serology the best choice. ${ }^{1}$ In our patient's case, the detection of Jamestown Canyon virus and Snowshoe hare virus IgM in his CSF, the identification of Jamestown Canyon virus-specific antibodies in the serum samples and the associated CSF pleocytosis support infection with Jamestown Canyon virus as the cause of his symptoms.

It is important to note that Jamestown Canyon virus and Snowshoe hare virus IgM may persist for more than a year in serum, so a single positive result does not necessarily indicate the presence of acute infection. ${ }^{13}$ In addition, multiple exposures to California serogroup viruses may result in reduced IgM responses. In certain cases of severe illness, a combination of IgM and plaque reduction neutralization testing on sera should be considered when California serogroup virus IgM is not initially found. ${ }^{4}$

Neuroinvasive infections due to Jamestown Canyon virus are infrequently reported in Canada, despite data supporting that a large part of the population is at risk for exposure. The paucity of reported cases is likely related, at least in part, to a large number of infections being subclinical or presenting with nonspecific symptoms, such as fever, that resolve spontaneously. However, a general lack of awareness of the epidemiology and clinical manifestations of infection among health care providers may contribute to the low number of reported cases. For patients who present with aseptic meningitis or encephalitis between April and October, consideration should be given to testing for California serogroup viruses, particularly when another cause (e.g., West Nile virus, Herpesvirus, enteroviruses) has not been clearly identified.

\section{References}

1. Drebot MA. Emerging mosquito-borne bunyaviruses in Canada. Can Commun Dis Rep 2015;41:117-23.

2. Pastula DM, Johnson DKH, White JL, et al. Jamestown Canyon virus disease in the United States - 2000-2013. Am J Trop Med Hyg 2015;93:384-9.

3. Goff G, Whitney H, Drebot MA. Roles of host species, geographic separation, and isolation in the seroprevalence of Jamestown Canyon and Snowshoe Hare viruses in Newfoundland. Appl Environ Microbiol 2012;78:6734-40.

4. Webster D, Dimitrova K, Holloway K, et al. California serogroup virus infection associated with encephalitis and cognitive decline, Canada, 2015. Emerg Infect Dis 2017;23:1423-4.

5. Krow-Lucal E, Lindsey NP, Lehman J, et al. West Nile virus and other nationally notifiable arboviral diseases - United States, 2015. MMWR Morb Mortal Wkly Rep 2017;66:51-5.

6. Human Jamestown Canyon virus infection - Montana, 2009. MMWR Morb Mortal Wkly Rep 2011;60:652-5.

7. West Nile virus and other mosquito-borne disease national surveillance report. 2016 - final - summary West Nile virus in Canada. Ottawa: Public Health Agency of Canada; 2016. Available: https://www.canada.ca/en/public-health/ services/diseases/west-nile-virus/west-nile-virus-other-mosquito-borne-disease. html (accessed 2017 Oct. 2).

8. Patriquin G, Schleihauf E, Johnston BL, et al. Seroprevalence of Jamestown Canyon virus in Nova Scotia [abstract]. Proceedings of the AMMI Canada - CACMID Annual Conference; 2016 Mar. 30-Apr. 2; Vancouver. J Assoc Med Microbiol Dis Can 2016;1:6-7. Available:www.cacmid.ca/wp-content/uploads/2016_CACMID_AMMI_Abstracts.pdf (accessed 2017 July 31).

9. Sampasa-Kanyinga $\mathrm{H}$, Levesque $\mathrm{B}$, Anassour-Laouan-Sidi E, et al. Zoonotic infections in communities of the James Bay Cree territory: an overview of seroprevalence. Can J Infect Dis Med Microbiol 2013;24:79-84.

10. Makowski K, Dimitrova K, Andonova M, et al. An overview of California serogroup virus diagnostics and surveillance in Canada in 2008. Int J Antimicrob Agents 2009;34:S19.

11. Deibel R, Grimstad PR, Mahdy MS, et al. Jamestown Canyon virus: The etiologic agent of an emerging human disease? Prog Clin Biol Res 1983;123:313-25.

12. Huang C, Campbell W, Grady L, et al. Diagnosis of Jamestown Canyon encephalitis by polymerase chain reaction. Clin Infect Dis 1999;28:1294-7.

13. Drebot M, Makowski K, Dimitrova K, et al. IgM persistence in probable cases of California serogroup infection. Am J Trop Med Hyg 2010;83:263.

\section{Competing interests: None declared.}

This article has been peer reviewed.

The authors have obtained patient consent.

Affiliations: Department of Neurology (Vosoughi), Department of Medical Microbiology \& Infectious Diseases (Walkty, Drebot, Kadkhoda) and Department of Immunology (Kadkhoda), Max Rady College of Medicine, University of Manitoba; Diagnostic Services Mani- toba (Walkty); National Microbiology Laboratory (Drebot); Cadham Provincial Laboratory (Kadkhoda), Winnipeg, Man.

Contributors: Reza Vosoughi and Andrew Walkty were involved in the initial draft of the case report. Andrew Walkty, Michael Drebot, and Kamran Kadkhoda all contributed equally to the initial draft of the discussion. All of the authors were involved in revising the manuscript, approved the final version to be published and agreed to act as guarantors of the work.

Acknowledgements: The authors thank Kristina Dimitrova and Nicole Barairo at the National Microbiology Laboratory for their technical assistance with this case.

Correspondence to: Andrew Walkty, awalkty@dsmanitoba.ca 